\title{
Mutations in the D1 domain of von Willebrand factor impair their propeptide-dependent multimerization, intracellular trafficking and secretion
}

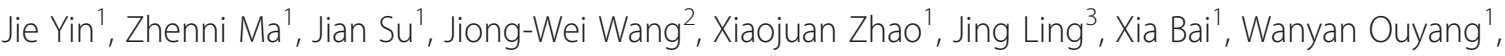 \\ Zhaoyue Wang ${ }^{1}$, Ziqiang $\mathrm{Yu}^{1}$ and Changgeng Ruan ${ }^{* *}$
}

\begin{abstract}
We identified three novel mutations (p.Gly39Arg, p.Lys157Glu, p.Cys379Gly) and one previously known mutation (p.Asp141Asn) in the von Willebrand factor propeptide from three von Willebrand disease patients. All four mutations impaired multimerization of von Willebrand factor, due to reduced oxidoreductase activity of isomeric propeptide. These mutations resulted in the endothelial reticulum retention and impaired basal and stimulated secretions of von Willebrand factor. Our results support that the mutations in the D1 domain lead to defective multimerization, intracellular trafficking, and secretion of von Willebrand factor and result in bleeding of patients.
\end{abstract}

Keywords: von Willebrand disease, WWF propeptide, WWF gene mutation, WWF multimerization, ER retention

\section{Findings}

Von Willebrand factor propeptide (VWFpp), composed of D1 and D2 domains, is necessary for the multimerization, intracellular trafficking, and secretion of the factor [1-3]. Few mutations in the D1 domain of VWFpp have been reported, and the pathogenic nature of these mutations remains largely unknown $[4,5]$.

We found three novel mutations (p.Gly39Arg, p.Lys157Glu, p.Cys379Gly) and one previously known mutation (p.Asp141Asn) in the VWFpp from three von Willebrand disease (VWD) patients (Additional file 1). The proband 1 (P1) and proband 2 (P2) were type 3 VWD, while the proband 3 (P3) was type 1 VWD [6]. The laboratory results of these patients and their family members are summarized in Table 1 . P1 and P2 presented undetectable von Willebrand factor (VWF) multimer, and P3 exhibited light multimer pattern, compared to normal plasma (NP) (Additional file 2: Figure S1). All mutations are located in the D1 domain of VWFpp.

\footnotetext{
* Correspondence: cshcma@medmail.com.cn

${ }^{1}$ Collaborative Innovation Center of Hematology, MOH Key Lab of Thrombosis and Hemostasis, Jiangsu Institute of Hematology, the First Affiliated Hospital, Soochow University, Suzhou 215006, China

Full list of author information is available at the end of the article
}

To determine whether and how these mutations impair VWF expression and function, we transiently transfected the human embryonic kidney 293 cells (HEK293) with wild type (WT) or VWF mutant constructs and analyzed VWF multimer (Fig. 1a,b). In the supernatant, the WT-VWF showed a full range of multimers similar to NP. Asp141Asn, Lys157Glu, and Cys379Gly mutants each exhibited different degrees of the loss of largeand medium-sized multimers. We also co-transfected Asp141Asn and Gly39Arg mutants and found that a partial VWF protein multimerization was readily detectable. When mutant and WT-VWF were cotransfected into HEK293 cells, the abnormal VWF multimers were all restored (Additional file 3: Figure S2). We examined cell lysates for VWF multimer and found that multimerization from all VWF mutations were inhibited in different levels as showed in the media. To determine the underlying mechanisms of defective VWF multimerization, we employed a previously established dimerization model using D1D2D'D3 fragment of VWF $[7,8]$. In this model, the decreased dimerization of D1D2D'D3 indicates the reduced oxidoreductase of mutant VWFpp. Under the non-reducing condition (Fig. 1c), truncated WT (T-WT) VWF formed both D'D3 monomers and dimers in the 
Table 1 Phenotypic and genetic features of three unrelated WWD families

\begin{tabular}{|c|c|c|c|c|c|c|c|c|c|c|}
\hline Family & Members & WF mutation & Genotype & $\begin{array}{l}\text { APTT ratio } \\
\text { (sec) }\end{array}$ & $\begin{array}{l}\text { PT ratio } \\
\text { (sec) }\end{array}$ & $\begin{array}{l}\text { TT ratio } \\
\text { (sec) }\end{array}$ & $\begin{array}{l}\text { WWF: Ag } \\
(\mathrm{IU} / \mathrm{dL})\end{array}$ & $\begin{array}{l}\text { WWF: RCo } \\
\text { (IU/dL) }\end{array}$ & $\begin{array}{l}\text { FVIII: C } \\
(\mathrm{IU} / \mathrm{dL})\end{array}$ & BS \\
\hline F1 & Proband P1 & G39R D141N & Compound heterozygous & 2.24 & 1.07 & 0.97 & 1.0 & 2.3 & 2.0 & 6 \\
\hline F1 & Father P1F & D141N & Heterozygous & 1.09 & 1.15 & 1.24 & 48.4 & 50.2 & 73.5 & 0 \\
\hline $\mathrm{F} 1$ & Mother P1M & G39R & Heterozygous & 1.12 & 1.05 & 0.91 & 30.3 & 36.4 & 50.6 & 1 \\
\hline F2 & Proband P2 & K157E C1165R & Compound heterozygous & 1.95 & 1.04 & 0.89 & 3.0 & 2.1 & 3.0 & 6 \\
\hline F2 & Father P2F & K157E & Heterozygous & 0.94 & 1.02 & 0.98 & 87.2 & 80.3 & 97.8 & 1 \\
\hline F3 & Proband P3 & C379G & Heterozygous & 1.50 & 1.17 & 1.07 & 8.0 & 5.9 & 13.1 & 9 \\
\hline F3 & Brother P3B & C379G & Heterozygous & 1.21 & 1.00 & 0.90 & 14.3 & 13.6 & 25.3 & 3 \\
\hline Normal range & - & - & - & $0.82-1.18$ & $0.86-1.14$ & $0.80-1.20$ & $50-160$ & $50-120$ & $50-150$ & $0-3$ or $0-5^{a}$ \\
\hline
\end{tabular}

PT prothrombin time, APTT activated partial thromboplastin time, $\pi$ thrombin time, VWF:Ag, von Willebrand antigen, VWF:RCo von Willebrand factor ristocetin cofactor activity, FVIII:C factor VIII coagulant activity, BS bleeding scores

${ }^{a} 0-3$ in male, $0-5$ in female
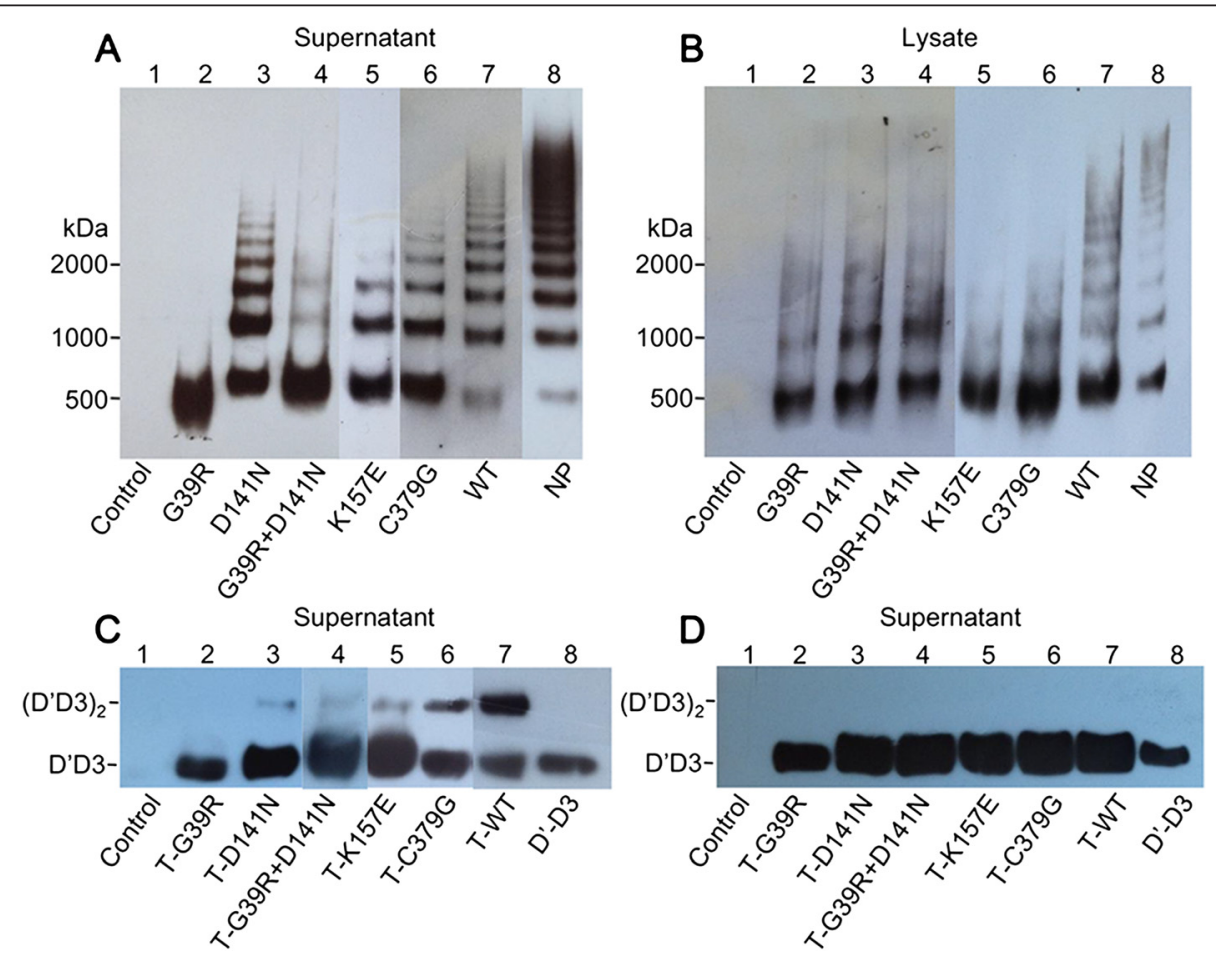

Fig. 1 Defective multimerization of mutant WWF and reduced dimerization of mutant truncated WWF. a HEK293 cells were transiently transfected by equal WT or mutant full-length WWF plasmid. Seventy hours after transfection, the supernatant of cells were collected for multimer analysis. In order to detect whether the mutations have abnormal multimer pattern, we measured equal WWF antigen of the mutations, wild type, and NP. Lane 1 is the negative control of the mimic empty vector. Lanes $2-6$ show the supernatant obtained from WWF mutant transfections and indicate decreases in high to medium-sized multimers to varying degrees. Exclusively, dimers formed with the Gly39Arg mutant (lane 2). The ability to form multimers was partly recovered by co-transfection of Gly39Arg and Asp141Asn (lane 4). b In the lysates of transfected cells, a similar decrease in the formation of WWF multimers was seen in the variants. However, WT-WWF exhibited a multimer pattern as observed for normal plasma. c HEK293 cells were transiently transfected by equal WT or mutant truncated WWF. In the media of transfected cells, dimerizations of truncated recombinant WWF were detected by Western blotting under the non-reducing condition. Lane 1 shows the empty pSecTag2/Hygro B vector. Dimers were absent in the Gly39Arg mutant (lane 2) while lanes 3-6 tapered in the dimer to different degrees. $\mathbf{d}$ In the supernatant of the transfected cells, the truncated WWF mutations and WT D1D2D'D3 fragment were examined under the reducing condition, and only D'D3 monomers were found 
supernatant. The dimerizations of truncated four variants (TGly39Arg, T-Asp141Asn, T-Lys157Glu, and T-Cys379Gly) were reduced, compared to that observed with T-WT. Cotransfection of the T-Gly39Arg and T-Asp141Asn resulted in a modest restoration of dimer formation compared to the single transfection of truncated Gly39Arg. This result indicates that the impaired multimerization is caused by decreased oxidoreductase activity of the isomeric propeptide. Under the reducing condition (Fig. 1d), D' D3 dimers were reduced to D'D3 monomers in both mutant and wild type D1D2D'D3 fragment.

The VWF antigen was measured in the conditioned media and cell lysates of the transiently transfected HEK293 cells (Additional file 4: Figure S3). The expressions of mutant Gly39Arg or Asp141Asn in the supernatant were significantly impaired, at the level of $2.8 \pm$ $0.3 \%$ to $2.5 \pm 0.1 \%$ of the WT. However, the expressions of mutant Lys157Glu or Cys379Gly product in the supernatant were less severely decreased at the levels of $26.0 \pm 4.1 \%$ and $22.4 \pm 3.8 \%$ of the WT. In co- transfection of Gly39Arg and Asp141Asn constructs, only $2.6 \pm 0.1 \%$ VWF was detectable in the supernatant, implying that both mutants do not mutually rescue each other from their defects. We also co-transfected the WT and each mutant (Gly39Arg, Asp141Asn, Lys157Glu, or Cys379Gly), and $59.3 \pm 4.3 \%, 57.3 \pm 8.9 \%, 86.6 \pm 7.1 \%$, or $33.9 \pm 1.2 \%$ of WT were detected, respectively. This suggests that four mutants can be partially restored by the WT. In the cell lysates of single or co-transfected variants, the VWF levels did not changed dramatically.

We found that the level of VWF antigen in the supernatant of HEK-293 cells expressing WT-VWF increased by 2.28-fold after phorbol 12-myristate-13-acetate (PMA) stimulation. However, cells expressing four mutations exhibited no significant changes in VWF antigen secretion upon PMA stimulation (Additional file 5: Figure S4).

By immunofluorescent staining of VWF proteins (Fig. 2a), we found that VWF formed green granules in cells. Only $37.8 \pm 9.3 \%$ WT-VWF was observed in the endoplasmic reticulum (ER), while $74.8 \pm 2.5 \%$ Gly39Arg,

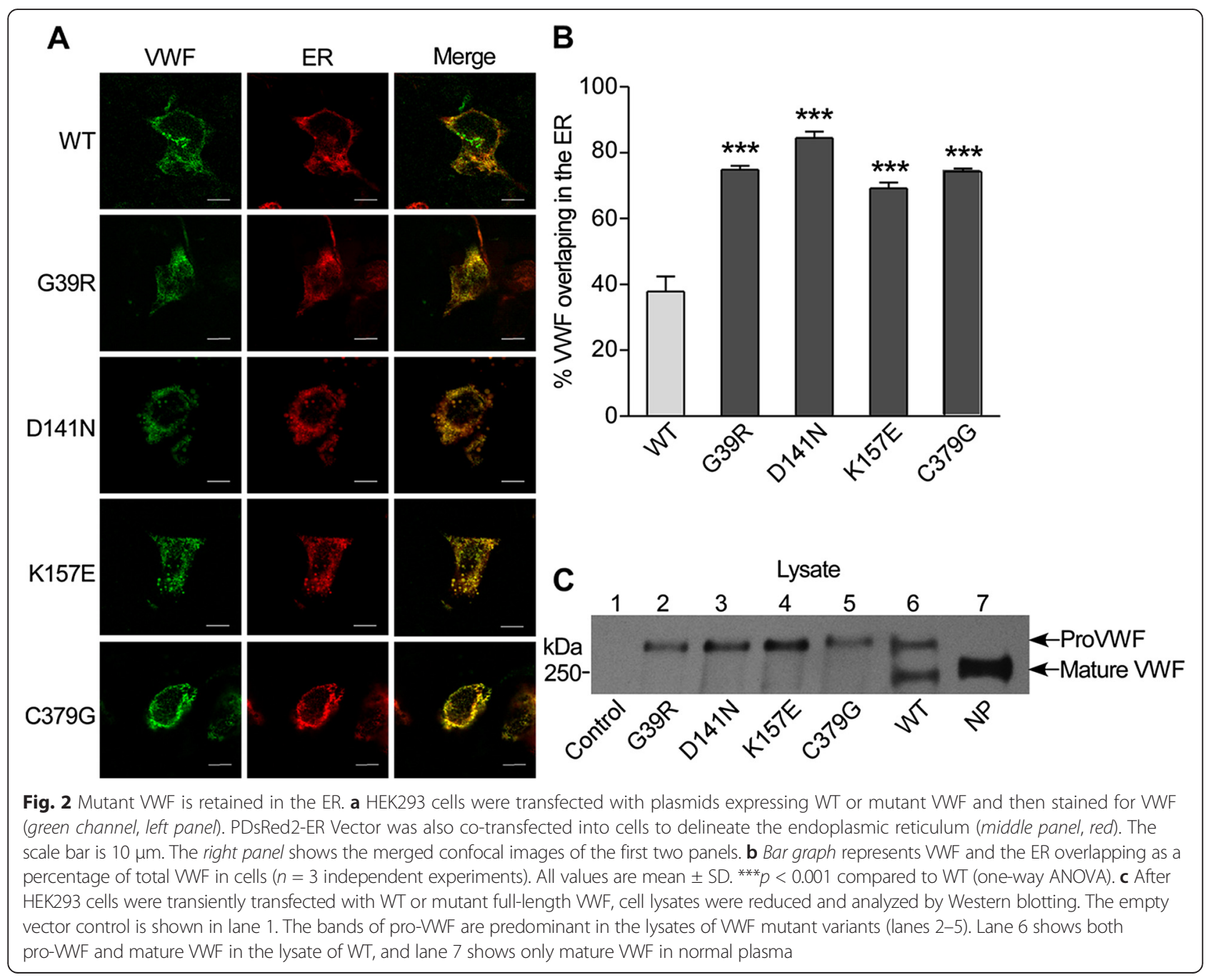


$84.6 \pm 3.8 \%$ Asp141Asn, $69.2 \pm 3.6 \%$ Lys157Glu, and $74.3 \pm 1.8$ \% Cys379Gly mutants were detected in the ER (Fig. 2b). In the cell lysates, the bands of pro-VWF were predominant, and mature VWF was hardly detectable in the cells expressing Gly39Arg, Asp141Asn, Lys157Glu, and Cys379Gly, compared to that of WT-VWF (Fig. 2c). These results indicate that the mutant VWF are retained in the ER and fail to be transported to the Golgi, resulting in the decreased VWF secretion.

In summary, four mutations in the D1 domain of VWF impair the multimerization of VWF by directly downregulating the oxidoreductase of the propeptide, disrupt the transport of VWF from the ER to the Golgi, and inhibit the basal and regulated secretion of VWF. These defects contribute to the quantitative loss of VWF, leading to the bleeding diathesis of VWD patients.

\section{Additional files}

Additional file 1: Supplementary data. Detailed methods and materials are shown.

Additional file 2: Figure S1. Plasma WWF multimers in three unrelated WWD patients and their family members. (A) Plasma samples were assessed by $1.3 \%$ SDS-agarose gel electrophoresis and Western blotting, and the image was taken after exposure for $3 \mathrm{~min}$. Normal plasma (NP) was shown in lane 4, 7 and 10 as a positive control. WW multimer structures were absent in lane 1 (P1) and lane 5 (P2), while P3 and P3B presented very light multimer bands. (B) The image was taken after 6-minute exposure. WWF multimer patterns of P3 and P3B were normal while those of P1 and P2 were still absent. (C) Schematic diagram of WWF. Arrows indicate the locations of four mutations in the D1 domain on the whole pro-WWF.

Additional file 3: Figure S2. Restored WWF multimerization of co-expression of mutant and WT-WWF. HEK293 cells were transiently transfected by WT and mutant full-length WWF plasmid in ratio of 1:1. WWF multimers present normal pattern in all co-expression of mutant and WT-WWF, compared to that observed with WT-WWF.

Additional file 4: Figure S3. Decreased basal secretion of mutant rWWF. HEK293 cells were transiently transfected by equal WT or mutant full-length WWF plasmid in single transfection. In co-transfection, two plasmids were transfected in ratio of 1:1, and added up to the equivalent plasmid of single transfection. WWF levels were determined in conditioned media and cell lysates from HEK293 cells transfected with single expressing vector or co-expressing vectors in duplicate. Each bar represents the average values of three separate transfections. WWF levels were measured by ELISA and are shown as percentages relative to WT-WF in media or lysates. The left side of the short vertical line represents single-transfections while the right side represents co-transfections.

Additional file 5: Figure S4. Decreased regulated secretion of mutant rWWF. Forty-eight hours post-transfection, HEK293 cells were rinsed and changed to release media with or without PMA (control). After further 60 min incubation, WWF levels were determined by ELISA in the release media and cell lysates. Each bar represents WWF secretion in the medium as a percentage of total WWF (medium plus lysate). Error bars indicate standard deviation of triplicate samples. The numbers above the bars indicate the fold increase of stimulated release compared with the control samples.

\section{Competing interests}

The authors declare that they have no competing interests.

\section{Authors' contributions}

$J Y$ and $C R$ were the principal investigators and took primary responsibility for the paper. ZM, JS, JW, JL, and WO performed the laboratory work for this study. XZ, XB, ZW, and ZY recruited the patients. JY and JW wrote the paper. All authors read and approved the final manuscript.

\section{Acknowledgments}

This work was supported by the Jiangsu Provincial Special Program of Medical Science (BL2012005), Jiangsu Province's Key Medical Center (ZX201102), Natural Science Foundation of Jiangsu Province (BK20140285), and the Priority Academic Program Development of Jiangsu Higher Education Institutions (PAPD). The authors thank Prof. Lijun Xia, Oklahoma Medical Research Foundation and Prof. Xiaosheng Wu, Mayo Clinic, for critical reading and revisions.

\section{Author details}

${ }^{1}$ Collaborative Innovation Center of Hematology, MOH Key Lab of Thrombosis and Hemostasis, Jiangsu Institute of Hematology, the First Affiliated Hospital, Soochow University, Suzhou 215006, China. ${ }^{2}$ Department of Surgery, National University of Singapore; Cardiovascular Research Institute (CVRI), National University Heart Centre Singapore (NUHCS), National University Health System, Singapore 999002, Singapore. ${ }^{3}$ Department of Hematology and Oncology, Children's Hospital of Soochow University, Suzhou 215003, China.

Received: 17 May 2015 Accepted: 3 June 2015 Published online: 20 June 2015

\section{References}

1. Journet AM, Saffaripour S, Wagner DD. Requirement for both D domains of the propolypeptide in von Willebrand factor multimerization and storage. Thromb Haemost. 1993;70(6):1053-7.

2. Rosenberg JB, Haberichter SL, Jozwiak MA, Vokac EA, Kroner PA, Fahs SA, et al. The role of the D1 domain of the von Willebrand factor propeptide in multimerization of WWF. Blood. 2002;100(5):1699-706.

3. Michaux G, Hewlett LJ, Messenger SL, Goodeve AC, Peake IR, Daly ME, et al. Analysis of intracellular storage and regulated secretion of 3 von Willebrand disease-causing variants of von Willebrand factor. Blood. 2003;102(7):2452-8. doi:10.1182/blood-2003-02-0599.

4. Baronciani L, Federici AB, Cozzi G, La Marca S, Punzo M, Rubini V, et al. Expression studies of missense mutations p.D141Y, p.C275S located in the propeptide of von Willebrand factor in patients with type 3 von Willebrand disease. Haemophilia. 2008;14(3):549-55.

5. Michiels JJ, Gadisseur A, van der Planken M, Schroyens W, Berneman Z. Laboratory and molecular characteristics of recessive von Willebrand disease type 2C (2A subtype IIC) of variable severity due to homozygous or double heterozygous mutations in the D1 and D2 domains. Acta Haematol. 2009;121(2-3):111-8.

6. Sadler JE, Budde U, Eikenboom JC, Favaloro EJ, Hill FG, Holmberg L, et al. Update on the pathophysiology and classification of von Willebrand disease: a report of the Subcommittee on von Willebrand Factor. J Thromb Haemost. 2006;4(10):2103-14.

7. Purvis AR, Sadler JE. A covalent oxidoreductase intermediate in propeptidedependent von Willebrand factor multimerization. J Biol Chem. 2004;279(48):49982-8.

8. Dang LT, Purvis AR, Huang RH, Westfield LA, Sadler JE. Phylogenetic and functional analysis of histidine residues essential for $\mathrm{pH}$-dependent multimerization of von Willebrand factor. J Biol Chem. 2011;286(29):25763-9.

\section{Abbreviations}

WD: von Willebrand disease; WF: von Willebrand factor; ER: endoplasmic reticulum; WFpp: WW propeptide; $\mathrm{P} 1$ : proband 1; $\mathrm{P} 2$ : proband 2 ; $\mathrm{P} 3$ : proband 3; NP: normal plasma; WT: wild type; PMA: Phorbol 12-myristate-13-acetate. 